\title{
Structural Design and Electrical Control of A New Rotary Joint Module Lina $\mathrm{Gu}^{\mathrm{a}}$, Guangjun Tian ${ }^{\mathrm{b}}$

\author{
School of Electrical Engineering, Yanshan University, Qinhuangdao066004, China; \\ agulinana@163.com, bt0208@126.com
}

Keywords: Ball screw; Three-linkmechanism; Rotary joint module; Electrical control.

\begin{abstract}
A new type of rotary joint module is put forward based on the principle of ball screw and the characteristic of three-link mechanism. First, the three-dimensional model of the joint module is built by SolidWorks. Second, the mathematical model of the joint module is established to do parameter design. And the driving performance of the joint module is analyzed based on the driving torque of the end-effector as object function. At last, the joint module is made, and a test platform of electrical control of the joint module is set up. The test shows that the rotational range of the joint module is larger so that the module has a wider application. Considered the mechanical structure, the module is more suitable for humanoid robot.
\end{abstract}

\section{Introduction}

At present, the drive modes of the rotary joint in humanoid robot are the hydraulic driving, the servo motor with harmonic gear drive, the pneumatic artificial muscle and the DC-motor with ball screw drive. The drive mode of the hydraulic driving has been widely applied. But its occupation space is large and its weight is heavy, so it is difficult to achieve the robot miniaturization [1]. For the second kind of drive mode, the required torque in the positive or reverse movement is different, so that the required driving force is very mismatch in the positive or reverse direction when a single motor drives the joint [2]. And the smooth movement of the robot is easily affected by quick stop or moving rapidly of the motor. The pneumatic artificial muscles is a typical flexible drive device with one-way, whose zooming motion is affected largely by the change of external load [3]. The drive mode of the DC-motor with ball screw is closer to the human muscle contraction which helps the human joint to complete any actions, and can provide greater torque $[4,5,6]$. The characteristics of this drive mode include the high deceleration capability, the transmitted torque with various direction and the instantaneous holding function on some level. It is feasible without the braking device in a limited loading condition so that this structure reduces energy consumption, saves manufacturing costs and reduces the mechanical body weight.

\section{Structural Design and Motion Analysis}

Structural Design of the Module. In this paper, based on the disadvantages of the existing rotary joint devices, a new type of rotary joint module combined the ball screw mechanism with the three-link mechanism is put forward, which is used in humanoid robot. As shown in Fig. 1, this two kinds of mechanisms are coupled together by sliding block so that the module can realize the transformation between the linear motion and rotation motion, variable speed drive, torque transfer efficiently. The connection type of screw thread is used in between modules which is simple and firm. 


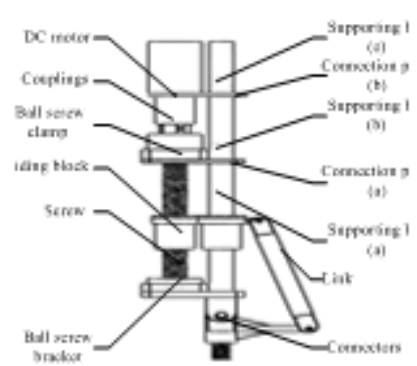

Fig. 1 Structure of the module
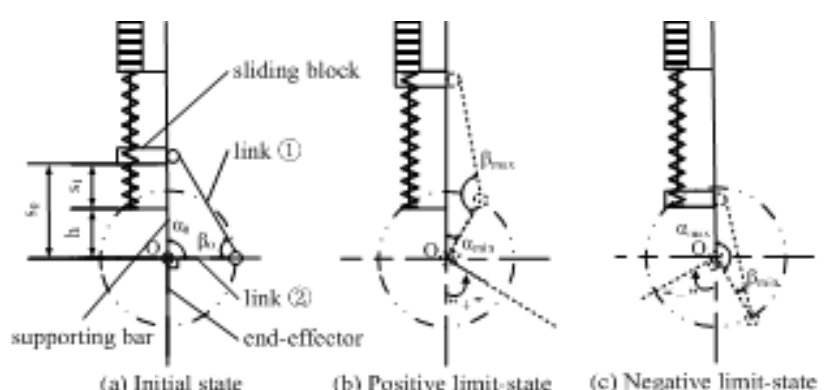

Fig. 2 Motion diagrams of the module

Motion Analysis for the Module. From the point of achieving the anticipated aim, we analyze the mechanical structure rationality. Three kinds of special motion states for the module are shown in the Fig. 2. Fig. 2(a) shows the initial state of the module that the state angle is $0^{\circ}$; Fig. 2(b) shows the limit-state of upward movement of the sliding block, instead of anti-clockwise motion of the end-effector, which is the position of maximum positive angle; Similarly, Fig. 2(c) shows the limit-state of downward movement of the sliding block, instead of clockwise motion of the end-effector, which is the position of maximum negative angle. The provision is based on its initial position in this paper, which the anti-clockwise is positive and the clockwise is negative.

The lengths of link (1) and link (2) are $l$ and $r$. The relational expression between them is

$l=m \cdot r$.

among formula (1): $m$ - the proportionality coefficient of the length of link (1) and link (2).

The $s_{0}$ is the distance from the sliding block to the rotational center $o$ in Fig. 2(a), based on the initial state, listed: $s_{0}=\sqrt{l^{2}-r^{2}}$, and $s_{0}=s_{1}+h$, the relational expression among $s_{1}, r$ and $m$ is

$$
s_{1}=r \sqrt{m^{2}-1}-h \text {. }
$$

among formula (2): $h$ - the distance between the end of screw and the rotational center $o$.

Due to the limited sections of this paper, other relational expressions can not be listed.

The ball screw of the module is SFK1202. When the displacement of sliding block is s, the desired rotational speed of motor is $n_{\mathrm{d}}=\mathrm{s} / 2(\mathrm{r})$. And the rated speed of motor is $N$, so that the required time $t$ can be calculated by

$$
t=(1 / N) \cdot(\mathrm{s} / 2)(\mathrm{s}) \text {. }
$$

So the relationship between the rotation angle $\theta$ and the displacement $\mathbf{s}$ can be expressed as

$$
\theta=\frac{\pi}{2}-\alpha=\frac{\pi}{2}-\arccos \left[\frac{r^{2}+\left(s_{0}+s\right)^{2}-l^{2}}{2 r\left(s_{0}+s\right)}\right] \text {. }
$$

\section{Parameters Calculation and Driving Performance Analysis}

Determine Sizes of Each Parts. The length of screw is initially selected to $160 \mathrm{~mm}$. Given the occupied space of other parts, the effective stroke of the sliding block can be calculated by $l_{\mathrm{e}}=75 \mathrm{~mm}$. Given the practical application, the range of anti-clockwise motion of the joint module is relatively larger. So $s_{1}$ is required to satisfy the following conditions:

$s_{1 \min } \leq s_{1} \leq l_{e} / 2$.

among formula (5): $s_{1 \min }$ - the minimum distance between the sliding block and the rotational center $o$.

Given the practical application and the occupied space of the joint module, based on formula (2) and (5), a set of parts sizes can be obtained to meet the demand of function design by the screening program of Matlab, which are $r=40 \mathrm{~mm}, m=2.5, l=m \cdot r=100 \mathrm{~mm}, s_{1}=27.65 \mathrm{~mm}, l_{\mathrm{e}}=75 \mathrm{~mm}, h=$ $64 \mathrm{~mm}$. According to the law of cosines, the limited angles among parts under different states of motion are listed in Table 1, referred to Fig. 2. The rotational range of the end-effector is $-57^{\circ} \sim+76.5^{\circ}$. 
Table 1 Angles among each parts under different motion states

\begin{tabular}{|c|c|c|c|c|}
\hline Angle & $\begin{array}{c}\text { The angle } \\
\text { between link (1) } \\
\text { and link (2) }\end{array}$ & $\begin{array}{l}\text { The angle between link } \\
\text { (2) and supporting bar }\end{array}$ & $\begin{array}{l}\text { The angular change } \\
\text { of the end-effector }\end{array}$ & $\begin{array}{l}\text { The rotational } \\
\text { range of the } \\
\text { end-effector }\end{array}$ \\
\hline $\begin{array}{c}\text { Clockwise } \\
\text { motion }\end{array}$ & $\beta_{\min }\left(20^{\circ}\right)$ & $\alpha_{\max }\left(147^{\circ}\right)$ & $\begin{array}{c}\alpha-\alpha_{\max }\left(-57^{\circ}\right) \\
\sim 0^{\circ}\end{array}$ & \multirow{3}{*}{$-57^{\circ} \sim+76.5^{\circ}$} \\
\hline Initial state & $\beta_{0}\left(66.5^{\circ}\right)$ & $\alpha_{0}\left(90^{\circ}\right)$ & & \\
\hline $\begin{array}{c}\text { Anti-clockwise } \\
\text { motion }\end{array}$ & $\beta_{\max }\left(160.5^{\circ}\right)$ & $\alpha_{\min }(13.5)$ & $\begin{array}{c}0^{\circ} \sim \\
\alpha-\alpha_{\min }\left(+76.5^{\circ}\right)\end{array}$ & \\
\hline
\end{tabular}

Driving Performance Analysis. In order to get the results of driving performance analysis, we analyze the links stress and the driving torque of the joint module [7]. Taking the upward movement of sliding block as an example, the stress analysis diagram of link (1) is shown in Fig. 3. Some uncertain factors need to be ignored in the process of analysis, such as friction. Assuming that the driving force of the sliding block in the movement remain unchanged, which is $F$, the driving torque of the joint module in the rotary motion is defined as

$$
M=F_{1} \cdot b=F \cdot\left(\frac{l^{2}+\left(s_{0}+s\right)^{2}-r^{2}}{2 l\left(s_{0}+s\right)}\right) \cdot r \cdot \sqrt{1-\left[\frac{l^{2}+r^{2}-\left(s_{0}+s\right)^{2}}{2 l \cdot r}\right]^{2}} .
$$

among formula (6):

$F_{1}$ - The driving force of the end-effector, $F_{1}=F \cdot \cos [\pi-(\alpha+\beta)]=F \cdot\left(\frac{l^{2}+\left(s_{0}+s\right)^{2}-r^{2}}{2 l\left(s_{0}+s\right)}\right)$;

$L$ - The driving force arm of the end-effector, $L=r \cdot \sin \beta=r \cdot \sqrt{1-\left[\frac{l^{2}+r^{2}-\left(s_{0}+s\right)^{2}-r^{2}}{2 l \cdot r}\right]^{2}}$.

Assuming that $\mathrm{F}=100 \mathrm{~N}$, according to formula (6), we get the diagram of driving performance analysis in Fig. 4. Through motion analysis of the module, we know that the relationship line is a approximate straight line between the angle $\beta$ and the displacement of the sliding block s. So the driving performance which is reflected by the driving torque is linear function with the displacement $\mathbf{s}$ or the angle $\beta$. And the angle $\alpha$ reflects indirectly the rotation angle $\theta$. For the driving performance maximization, the maximum point should be located in the middle of the rotational range of the end-effector. According to the section 3.2, the range of the rotation angle $\theta$ is $-57^{\circ} \sim+76.5^{\circ}$. Known by Fig. 4, the whole motion range of the joint module is almost averagely divided by the high point of the driving performance. The result shows this set of parts sizes is suitable and makes the joint module realize the driving performance maximization.

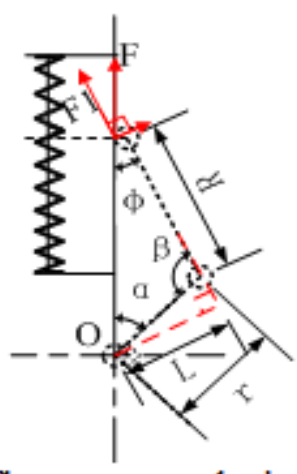

Fig. 3 The stress analysis graph

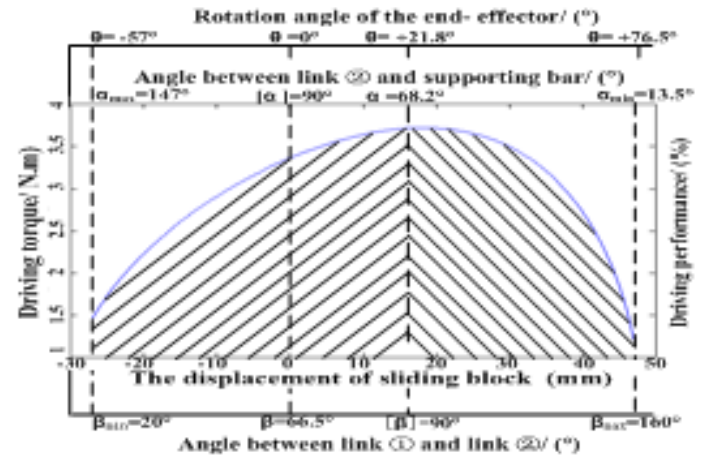

Fig. 4 The diagram of driving performance analysis

\section{Electrical Control of the Module}

The electrical control program of the module is mainly divided into the following four parts. The flow diagram of electrical control scheme is shown in Fig.5. 


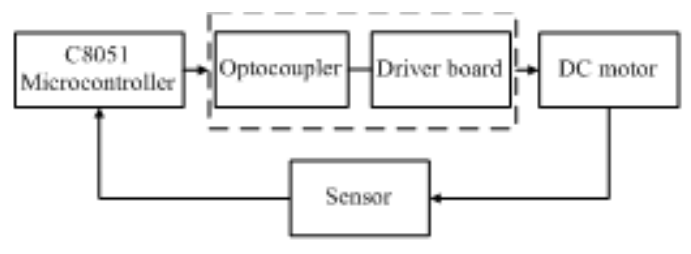

Fig. 5 Diagram of electrical control scheme

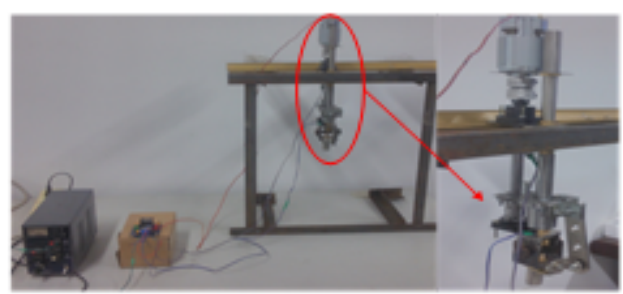

Fig. 6 The electrical control test

(1)Control section: From aspects of the performance, power consumption, economy, and the functional requirements of the module body design into consideration, the C8051 microcontroller is selected as the control core of the electrical control test to achieve the motor to start and stop control, reversing control, speed control.

(2)Drive part: The motor driver board LKV-HM3.0 is selected with small size, light weight, high driving power, and strong driving capability [8], whose driving chip is the L298N with dual H-bridge by ST company. And because the drive voltage of motor and the operating voltage of microcontroller are different, we need to add a optoelectric coupler to isolate voltage on both sides [9].

(3)Sensing part: There are two schemes for sensor selection. First, the distance between the sliding block and the top of module is measured by the displacement sensor to realize real-time distance measurement. we obtain the rotation angle of the end-effector through the relationship of the displacement of sliding block and the rotation angle. Second, the rotational angle is directly measured by the rotational angle sensor which can be installed on the end-effector of the joint module.

In order to avoid the motor locked-rotor phenomenon, we need to fit limit switches at the upper and lower limit position of the sliding block movement to ensure the safety of the motor running.

(4)Power section: In the test phase, the DC power is obtained by the switching power which mainly supplies rectifier, filter, regulator. In the future, the system realizes off-line movement with the battery power of $24 \mathrm{~V} / 12 \mathrm{~V}$.

Through three-dimensional modeling and theoretical analysis, we think the module is feasible to some extent. In order to further validate the feasibility of the joint module, the experimental prototype device is made, and the test platform is set up to achieve electrical control of the joint module, as shown in Fig. 6. The feasibility of structure design and control has been well validated. There are simple structure and large movement range to the joint module which can satisfy the requirements of rotation angle of human joint when human body is walking. The joint module can be used in humanoid robot.

\section{Conclusion}

A new type of rotary joint moduleis put forward, which realizes the transformation between the linear motion and rotation motion, variable speed drive and torque transfer efficiently. The drive mode of DC-motor with ball screw is selected for the joint module, which reduces the power requirements of the motor drive in the rotary joint. The structure of the joint module is simple, reliable and easy to implement. It is beneficial to simplify the combined mechanical structure with some versatility. Because its structure, movement, and drive mode are closer to the human joints, the joint module has great value in the study of humanoid robot.

\section{Acknowledgements}

This work was supported by the National Natural Science Foundation of China (Grant No. 51475407). The patent application for the rotary joint module has been accepted by the State Intellectual Property Office of P. R. China.

\section{References}

[1] Y.L. Han, S.Q. Zhu and B, China Patent 201310437171.6. (2014) 
[2] J. Wang, China Patent 201010233153.2. (2010)

[3] L.M. Sui, Z.W. Wang and G. Bao, Design of an anthropomorphic arm actuated by pneumatic muscles, Chinese Hydraulics and Pneumatic. 9 (2004) 7-8.

[4] M. Yang, C.B Yin, and Y.H. Dong, The research status of lower limbs of humanoid walking robot, Mechanical Drive. 30 (2006) 84-89.

[5] J.H. Fu, Mechanical design and walking simulation of humanoid Robot, Northeast University. (2010)

[6] S.B. Rok, Design of a redundantly actuated leg mechanism, Proceeding of the 2003 IEEE International Conference on Robotics \& Automation. (2003)

[7] D.X. Cheng ect, Handbook of Mechanical Design, third ed., Chemical Industry Publishing House, Beijing, 2008.

[8] ALSROBOT, User Manual of Drive Board to DC-motor with Double H-bridge V2.0, 2008.

[9] W.Z. Jia, J.P. Wang and Y.H. Kang, Design of motor speed/ current control system based on single chip, Value Engineering. (2016) 247-249. 